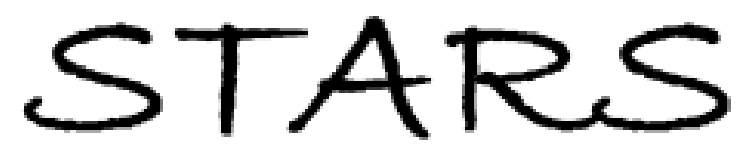

University of Central Florida

STARS

$1-1-2010$

\title{
Time-dependent density-matrix functional theory for biexcitonic phenomena
}

Volodymyr Turkowski

University of Central Florida

Carsten A. Ullrich

Talat S. Rahman

University of Central Florida

Michael N. Leuenberger

University of Central Florida

Find similar works at: https://stars.library.ucf.edu/facultybib2010

University of Central Florida Libraries http://library.ucf.edu

This Article is brought to you for free and open access by the Faculty Bibliography at STARS. It has been accepted for inclusion in Faculty Bibliography 2010 s by an authorized administrator of STARS. For more information, please contactSTARS@ucf.edu.

\section{Recommended Citation}

Turkowski, Volodymyr; Ullrich, Carsten A.; Rahman, Talat S.; and Leuenberger, Michael N., "Timedependent density-matrix functional theory for biexcitonic phenomena" (2010). Faculty Bibliography 2010s. 872.

https://stars.library.ucf.edu/facultybib2010/872

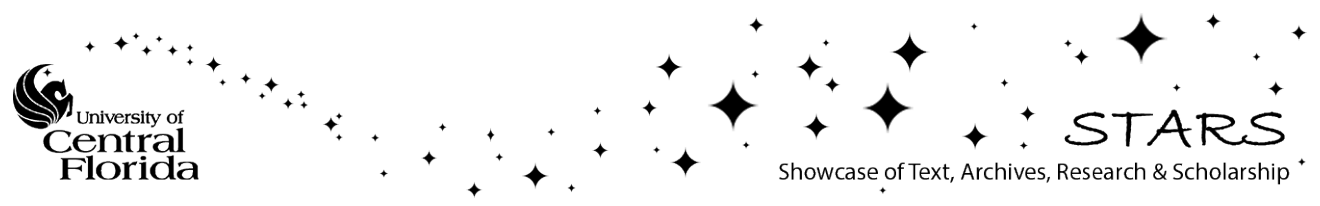




\title{
Time-dependent density-matrix functional theory for biexcitonic phenomena
}

\author{
Volodymyr Turkowski, ${ }^{1}$ Carsten A. Ullrich, ${ }^{2}$ Talat S. Rahman, ${ }^{1}$ and Michael N. Leuenberger ${ }^{1, *}$ \\ ${ }^{1}$ Department of Physics and NanoScience and Technology Center, University of Central Florida, Orlando, Florida 32816, USA \\ ${ }^{2}$ Department of Physics and Astronomy, University of Missouri, Columbia, Missouri 65211, USA
}

(Received 9 August 2010; published 17 November 2010)

\begin{abstract}
We formulate a time-dependent density-matrix functional theory (TDDMFT) approach for higher-order correlation effects like biexcitons in optical processes in solids based on a reduced two-particle density-matrix formalism within the normal orbital representation. A TDDMFT version of the Schrödinger equation for biexcitons in terms of one- and two-body reduced density matrices is derived, which leads to finite biexcitonic binding energies already with an adiabatic approximation. Biexcitonic binding energies for several bulk semiconductors are calculated using a contact biexciton model.
\end{abstract}

DOI: $10.1103 /$ PhysRevB.82.205208

PACS number(s): 71.10.-w, 71.15.Mb, 71.45.Gm

\section{INTRODUCTION}

The theoretical description of ultrafast processes in modern electronic devices is an important problem of contemporary condensed-matter physics. ${ }^{1,2}$ The entangled role of the fluctuation and correlation effects, especially in low dimensions, makes such an examination a challenge. In particular, it is not easy to reproduce correct excitonic and biexcitonic features in the optical absorption spectra of materials. Besides fundamental interest such as in four-wave mixing,,$^{3,4}$ excitonic and biexcitonic effects have a variety of practical applications, such as optoelectronic devices, ${ }^{5}$ entangled photon sources, ${ }^{6}$ and quantum computing. ${ }^{7}$

The standard approaches, based on the semiconductor Bloch equations (SBEs) (Ref. 8) and nonequilibrium Green's-function techniques, ${ }^{9,10}$ cannot be easily applied to study higher-order correlation effects in strongly nonequilibrium situation because this requires many-particle correlation functions that depend on many time arguments. ${ }^{11}$ Approaching these problems with time-dependent density-functional theory (TDDFT) (Ref. 12) looks promising due to its formal simplicity and the fact that it, in principle, includes correlation effects exactly; however, this usually requires going beyond the standard local-density and generalized gradient approximations (LDA-GGA). ${ }^{13}$ Excitonic effects have been studied with TDDFT in several ways, including the timedependent optimized effective potential approach ${ }^{14}$ and the combination with the Bethe-Salpeter method. ${ }^{1}$ Unfortunately, these approaches also become very tedious in the strongly nonequilibrium case.

Recently, we proposed an alternative approach for ultrafast excitonic effects based on the single-particle density matrix and a TDDFT version of the SBEs. ${ }^{15,16}$ We showed that the effective electron-hole attraction is defined by matrix elements of the exchange-correlation $(\mathrm{XC})$ kernel $f_{\mathrm{XC}}$ with respect to the valence and conduction band Kohn-Sham single-particle wave functions. An approximate $f_{\mathrm{XC}}$ based on the adiabatic LDA leads to a too weak electron-hole attraction to produce bound excitons. Instead, experimentally observed lowest exciton binding energies can be reproduced in a simple way using the local and long-range XC kernels

$$
f_{\mathrm{XC}}^{\text {local }}\left(\mathbf{r}, \mathbf{r}^{\prime}\right)=-A_{0} \delta\left(\mathbf{r}-\mathbf{r}^{\prime}\right)
$$

and

$$
f_{\mathrm{XC}}^{\mathrm{LR}}\left(\mathbf{r}, \mathbf{r}^{\prime}\right)=-\frac{\alpha}{\left|\mathbf{r}-\mathbf{r}^{\prime}\right|}
$$

where $A_{0}$ and $\alpha$ can be viewed as adjustable parameters.

The local XC kernel (1) can be interpreted ${ }^{13,17}$ as playing a similar role in TDDFT as the contact exciton model developed in the early seventies. ${ }^{18,19}$ Models of this kind typically produce a single bound exciton. Results of similar quality are obtained with the long-range kernel (2), despite its different structure. ${ }^{20,21}$ Both $f_{\mathrm{XC}}^{\text {local }}$ and $f_{\mathrm{XC}}^{\mathrm{LR}}$ produce optical spectra of semiconductors in reasonable agreement with experiment. $^{13,22}$

In the case of biexcitons, which are correlated double electronic excitations, the problem is much more complicated. One reason is that multiple excitations in TDDFT require nonadiabatic XC functionals, and so far there are no simple approximations available. Another reason is that at first sight it is not clear how to represent biexcitonic wave functions in Kohn-Sham TDDFT. In this paper, we formulate and test an alternative TDDMFT approach for biexcitons based on the natural orbital (NO) representation for the stationary electron eigenfunctions, ${ }^{23-25}$ where the multiparticle excited states are naturally related to the higher-order density-matrix elements.

\section{TDDMFT FORMALISM}

The standard TDDFT single-particle Hamiltonian is

$$
\hat{h}(\mathbf{r}, t)=-\frac{\nabla^{2}}{2}+V(\mathbf{r}, t)+V_{\mathrm{H}}[n](\mathbf{r}, t)+V_{\mathrm{XC}}[n](\mathbf{r}, t) .
$$

Here, $V(\mathbf{r}, t)=V_{\text {nucl }}(\mathbf{r})+V_{\text {ext }}(\mathbf{r}, t)$ is the static potential of the nuclei plus the time-dependent external perturbing potential. We consider a homogeneous external electric field in dipole approximation, $V_{\text {ext }}(\mathbf{r}, t)=-\mathbf{r} E(t)$, which implies that the characteristic field frequency is much larger than the level spacing. ${ }^{26} V_{\mathrm{H}}(\mathbf{r}, t)$ is the Hartree potential and $V_{\mathrm{XC}}(\mathbf{r}, t)$ is the time-dependent XC potential; both are functionals of the time-dependent single-particle density $n(\mathbf{r}, t)$. The Hartree potential is not very important for the description of excitons but the $\mathrm{XC}$ potential is crucial, since it accounts for the effective electron-hole interaction. ${ }^{16}$ 
In general, $V_{\mathrm{XC}}[n](\mathbf{r}, t)$ has a memory, i.e., it depends on densities at previous times $t^{\prime} \leq t$. The resulting XC kernel $f_{\mathrm{xc}}$, defined as

$$
f_{\mathrm{XC}}\left(\mathbf{r}, \mathbf{r}^{\prime}, \omega\right)=\left.\int d\left(t-t^{\prime}\right) e^{i \omega\left(t-t^{\prime}\right)} \frac{\delta V_{\mathrm{XC}}(\mathbf{r}, t)}{\delta n\left(\mathbf{r}^{\prime}, t^{\prime}\right)}\right|_{n_{0}(\mathbf{r})},
$$

therefore has in general a frequency dependence. An explicitly frequency-dependent $f_{\mathrm{XC}}$ is required for describing double excitations with linear-response TDDFT. ${ }^{27,28}$ However, to date there are only few nonadiabatic approximations for $f_{\mathrm{XC}}$ available, and none of them is particularly suited for the biexcitonic properties in solids we have in mind. For this reason, we choose a different approach.

To describe the properties of doubly excited $N$-electron systems, one can consider the one- and two-electron density matrices, defined as ${ }^{23-25}$

$$
\begin{aligned}
\gamma\left(x_{1}, x_{1}^{\prime}, t\right)= & N \int d x_{2} \int d x_{3} \ldots \int d x_{N} \\
\times & \Psi\left(x_{1}, x_{2}, \ldots, x_{N}, t\right) \Psi^{*}\left(x_{1}^{\prime}, x_{2}, \ldots, x_{N}, t\right),(5) \\
\Gamma\left(x_{1}, x_{2}, x_{1}^{\prime}, x_{2}^{\prime}, t\right)= & N(N-1) \int d x_{3} \int d x_{4} \ldots \int d x_{N} \\
& \times \Psi\left(x_{1}, x_{2}, \ldots, x_{N}, t\right) \Psi^{*}\left(x_{1}^{\prime}, x_{2}^{\prime}, \ldots, x_{N}, t\right),
\end{aligned}
$$

where $\Psi$ is the many-body wave function and $x_{i}=\left(\mathbf{r}_{i}, s_{i}\right)$ denotes the space coordinate and spin index. $\gamma\left(x_{1}, x_{1}^{\prime}, t\right)$ describes the single-particle properties of the system, such as the charge density $n(x, t)=\gamma(x, x, t)$. Moreover, all groundstate quantities, including $\Gamma_{0}\left(x_{1}, x_{2}, x_{1}^{\prime}, x_{2}^{\prime}\right)$, can, in principle, be obtained from $\gamma_{0}\left(x_{1}, x_{1}^{\prime}\right)$, since there is one-to-one correspondence between $\gamma_{0}\left(x_{1}, x_{1}^{\prime}\right)$ and the ground-state manybody wave function $\Psi_{0}$ (the DMFT generalization of the Hohenberg-Kohn theorem ${ }^{29}$.

Let us now restrict the discussion to two-electron systems and derive equations of motion for the density matrices. We consider the following effective two-electron Hamiltonian:

$$
\hat{H}\left(\mathbf{r}_{1}, \mathbf{r}_{2}, t\right)=\hat{h}^{\mathrm{ad}}\left(\mathbf{r}_{1}, t\right)+\hat{h}^{\mathrm{ad}}\left(\mathbf{r}_{2}, t\right)+w\left[n_{2}\right]\left(\mathbf{r}_{1}, \mathbf{r}_{2}, t\right),
$$

where $\hat{h}^{\text {ad }}$ is the TDDFT Hamiltonian (3) using an adiabatic approximation for $V_{\mathrm{XC}}[n](\mathbf{r}, t)$, which leads to a frequencyindependent XC kernel $f_{\mathrm{XC}}^{\mathrm{ad}}\left(\mathbf{r}, \mathbf{r}^{\prime}\right)$. In this way, excitons can still be described since the frequency dependence of $f_{\mathrm{XC}}$ is not essential for the electron-hole interaction. However, biexcitons (which are correlated two-particle excitations) cannot be captured in the adiabatic approximation. To make up for this, we introduce, in a somewhat ad hoc manner, an effective two-particle interaction $w\left[n_{2}\right]\left(\mathbf{r}_{1}, \mathbf{r}_{2}, t\right)$ which we define as a functional of the two-particle density $n_{2}\left(\mathbf{r}_{1}, \mathbf{r}_{2}, t\right)$ $=\Psi^{*}\left(\mathbf{r}_{1}, \mathbf{r}_{2}, t\right) \Psi\left(\mathbf{r}_{1}, \mathbf{r}_{2}, t\right)$. In this way, dynamical screening effects can in principle be accounted for, as done in standard many-body perturbation theory.

In the following we express the two-electron wave function in terms of the NOs $\chi_{k}(\mathbf{r})$. In the singlet case one obtains $\Psi\left(\mathbf{r}, \mathbf{r}^{\prime}, t\right)=\Sigma_{k, l} C_{k l}(t) \chi_{k}(\mathbf{r}) \chi_{l}\left(\mathbf{r}^{\prime}\right)$, where $C_{k l}(t)$ is a symmetric matrix, and $k, l$ are the appropriate quantum numbers (band index, momentum, spin, etc.). We shall use this matrix in general nondiagonal form for physical insight on the nature of the excitations. Since the density matrices $\gamma\left(x_{1}, x_{1}^{\prime}, t\right)$ and $\Gamma\left(x_{1}, x_{2}, x_{1}^{\prime}, x_{2}^{\prime}, t\right)$ are defined by the two-electron wave function, they can be expressed in terms of the matrix elements $C_{k l}(t)$,

$$
\begin{gathered}
\gamma\left(x_{1}, x_{1}^{\prime}, t\right)=\sum_{k, l} \gamma_{k l}(t) \chi_{k}\left(x_{1}\right) \chi_{l}^{*}\left(x_{1}^{\prime}\right), \\
\Gamma\left(x_{1}, x_{2}, x_{1}^{\prime}, x_{2}^{\prime}, t\right)=\sum_{k l m n} \Gamma_{k l m n}(t) \chi_{k}\left(x_{1}\right) \chi_{l}\left(x_{2}\right) \chi_{m}^{*}\left(x_{1}^{\prime}\right) \chi_{n}^{*}\left(x_{2}^{\prime}\right),
\end{gathered}
$$

where $\gamma_{k l}(t)=2 \Sigma_{m} C_{k m}(t) C_{l m}^{* T}(t)$ and $\Gamma_{k l m n}(t)=2 C_{k l}(t) C_{m n}^{*}(t)$. We will soon see that in the two-band approximation the excitonic wave function is proportional to $\gamma_{\mathbf{k}_{1} \mathbf{k}_{2}}^{c v}(t)$, and the biexcitonic one to $\Gamma_{\mathbf{k}_{1} \mathbf{k}_{2} \mathbf{k}_{3} \mathbf{k}_{4}}^{c c v v}(t)$, where $c$ and $v$ stand for the conduction and valence bands, and $\mathbf{k}_{i}$ is the corresponding electron and hole momentum. From now on, we shall use superscripts to denote band indices.

The equation of motion for $\gamma_{k l}(t)$ and $\Gamma_{k l m n}(t)$ can be obtained via the equation for $C_{k l}(t)$. From the time-dependent two-electron Schrödinger equation one finds

$$
i \frac{\partial C_{k l}(t)}{\partial t}=\sum_{r}\left[h_{k r}(t) C_{r l}(t)+C_{k r}(t) h_{l r}(t)\right]+\sum_{r s} w_{k l r s}(t) C_{r s}(t)
$$

with the initial condition $C_{k l}(t=0)=\delta_{k l} c_{k}$ and the matrix elements

$$
\begin{gathered}
h_{k r}(t)=\int d \mathbf{r} \chi_{k}^{*}(\mathbf{r}) \hat{h}^{\mathrm{ad}}(\mathbf{r}, t) \chi_{r}(\mathbf{r}), \\
w_{k l m n}(t)=\int d \mathbf{r}_{1} \int d \mathbf{r}_{2} \chi_{k}^{*}\left(\mathbf{r}_{1}\right) \chi_{l}^{*}\left(\mathbf{r}_{2}\right) w\left[n_{2}\right]\left(\mathbf{r}_{1}, \mathbf{r}_{2}, t\right) \\
\quad \times \chi_{m}\left(\mathbf{r}_{1}\right) \chi_{n}\left(\mathbf{r}_{2}\right) .
\end{gathered}
$$

Here and in the following, it is implied that each spatial integration is divided by the unit cell volume. Equation (10) is nonlinear, since the matrix elements, which depend on the electron density, are functions of $C_{k l}(t)$. From the definitions of $\gamma_{k l}(t)$ and $\Gamma_{k l m n}(t)$ one then obtains the following equations for the one- and two-particle matrix elements:

$$
\begin{gathered}
i \frac{\partial \gamma_{k l}}{\partial t}=\sum_{r}\left(h_{k r} \gamma_{r l}-\gamma_{k r} h_{r l}\right)+\sum_{r, s, m}\left(\Gamma_{l r s m}^{*} w_{m s r k}^{*}-\Gamma_{k r s m} w_{m s r l}\right), \\
i \frac{\partial \Gamma_{k l m n}}{\partial t}=\sum_{r}\left(h_{k r} \Gamma_{r l m n}+h_{r l} \Gamma_{k r m n}-h_{r m} \Gamma_{k l r n}-h_{r n} \Gamma_{k l m r}\right) \\
+\sum_{r, s}\left(w_{k l r s} \Gamma_{r s m n}-w_{m n r s}^{*} \Gamma_{k l r s}\right) .
\end{gathered}
$$

An important feature of Eqs. (13) and (14) is the fact that they are closed, i.e., one does not need to truncate an infinite hierarchy of equations for higher-order density-matrix ele- 
ments. However, keep in mind that this property is only valid for two-level (two-band) systems. In the single-electron $(w=0)$ linearized diagonal approximation for two bands, one obtains the TDDFT-Wannier equation for the exciton eigenenergies and eigenfunctions from Eq. (7), ${ }^{16}$

$$
E_{n \mathbf{q}}^{v} \gamma_{n \mathbf{k}, \mathbf{q}}^{c v}=\sum_{\mathbf{k}^{\prime}}\left[\left(\varepsilon_{\mathbf{k}^{\prime}+\mathbf{q}}^{c}-\varepsilon_{\mathbf{k}^{\prime}}^{v}\right) \delta_{\mathbf{k k}^{\prime}}+F_{\mathbf{k k}^{\prime}}\right] \gamma_{n \mathbf{k}^{\prime}, \mathbf{q}}^{c v}
$$

where $\mathbf{k}$ is the electron momentum, $\mathbf{q}$ is the sum of the electron and hole momenta (the exciton momentum) and

$$
F_{\mathbf{k k}^{\prime}}=2 \int d \mathbf{r} \int d \mathbf{r}^{\prime} \chi_{c \mathbf{k}}^{*}(\mathbf{r}) \chi_{v \mathbf{k}}(\mathbf{r}) f_{\mathrm{xc}}\left(\mathbf{r}, \mathbf{r}^{\prime}\right) \chi_{v \mathbf{k}^{\prime}}^{*}\left(\mathbf{r}^{\prime}\right) \chi_{c \mathbf{k}^{\prime}}\left(\mathbf{r}^{\prime}\right)
$$

are the matrix elements for the effective electron-hole attraction.

\section{TWO-LEVEL MODEL FOR BIEXCITONS}

The possibility to obtain a biexcitonic state with the TDDMFT formalism can be already shown for a two-level model with the energy levels $E_{1}$ and $E_{2}>E_{1}$ and the highest occupied molecular orbital-lowest unoccupied molecular orbital gap $E_{g}=E_{2}-E_{1}$. From Eqs. (13) and (14) in the lowest(second-) order approximation (by keeping only those matrix elements which contain no more than two indices " 2 "), one obtains the following system of equations after carrying out a Fourier transformation into the frequency domain,

$$
\begin{aligned}
& \left(\omega-E_{g}-F^{1}\right) \gamma^{21}-G^{1} \Gamma^{2211}=0, \\
& \left(\omega-2 E_{g}-G^{2}\right) \Gamma^{2211}-F^{2} \gamma^{21}=0,
\end{aligned}
$$

where $F^{1}=w_{2121}-w_{1111}+w_{2112}+h_{21}^{\gamma}+w_{2111}^{\gamma}, F^{2}=2 h_{21}+w_{2212}$ $+w_{2221}+w_{2211}^{\gamma}, \quad G^{1}=h_{12}+w_{2122}-w_{1112}+w_{2111}^{\Gamma}, \quad G^{2}=w_{2222}$ $-w_{1111}+w_{2211}^{\Gamma}$, and

$$
\begin{gathered}
h_{a b}^{\gamma}=\int d 1 d 2 \chi_{a}^{*}(1) \chi_{b}(1) \frac{\delta V_{\mathrm{XC}}(1)}{\delta n(2)} \chi_{2}(2) \chi_{1}^{*}(2), \\
w_{a b c d}^{\gamma}=\int d 1 d 2 d 3 \chi_{a}^{*}(1) \chi_{b}^{*}(2) \frac{\delta w(1,2)}{\delta n(3)} \\
\quad \times \chi_{c}(1) \chi_{d}(2) \chi_{2}(3) \chi_{1}^{*}(3), \\
w_{a b c d}^{\Gamma}=\int d 1 d 2 d 3 d 4 \chi_{a}^{*}(1) \chi_{b}^{*}(2) \frac{\delta w(1,2)}{\delta n(3,4)} \\
\times \chi_{c}(1) \chi_{d}(2) \chi_{2}(3) \chi_{2}(4) \chi_{1}^{*}(3) \chi_{1}^{*}(4) .
\end{gathered}
$$

All matrix elements are evaluated at the initial (nonperturbed) densities, and we use the shorthand notation 1 for $\mathbf{r}_{1}$, 2 for $\mathbf{r}_{2}$, etc. The solutions of the system of Eq. (17) can be easily found

$$
\omega=\frac{3 E_{g}+F^{1}+G^{2}}{2} \pm \frac{1}{2} \sqrt{\left(E_{g}-F^{1}+G^{2}\right)^{2}+4 G^{1} F^{2}} .
$$

From the general solution, one can discuss several limiting cases. In particular, for no correlations $\left(F^{1}=F^{2}=G^{1}\right.$

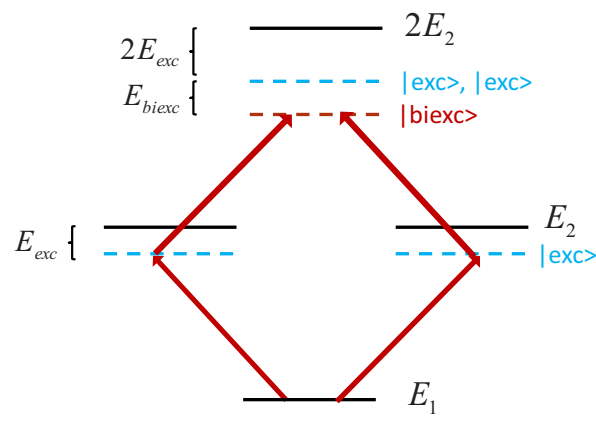

FIG. 1. (Color online) Excitonic bound states in a two-level system with single-particle energies $E_{1}$ and $E_{2} . E_{\text {exc }}$ and $E_{\text {biexc }}$ are the binding energies of excitons and biexcitons, respectively. A biexcitonic state can be thought of as arising from a two-step process: First, two excitons are created, which then combine to form a biexciton whose energy is less than the energy of the two individual excitons.

$=G^{2}=0$ ) one gets a trivial solution with one and two free excited electrons: $\omega_{1}=E_{g}, \omega_{2}=2 E_{g}$. In the absence of a density-dependent two-electron potential $\left(w\left[n_{2}\right]\left(\mathbf{r}_{1}, \mathbf{r}_{2}\right)=G^{2}\right.$ $=0$ ), one finds an excitonic state with energy $\omega_{1}=E_{g}-F^{1}$ $\approx E_{g}-E_{\text {exc }}<E_{g}$ (at large gap). However, the second root $\omega_{2}$ cannot be lower than $2 E_{g}-2 E_{\text {exc }}$, i.e., the linear adiabatic approximation does not produce a biexciton, as expected. On the other hand, for nonzero $w\left[n_{2}\right]\left(\mathbf{r}_{1}, \mathbf{r}_{2}\right)$, when $G^{2}<0$ and $\left|F^{1}\right|<\left|G^{2}\right|$, one can obtain a biexcitonic level with binding energy $\approx G^{2}-2 F^{1}$.

Therefore, in order to obtain a biexcitonic state in pure TDDFT $\left\{\right.$ with no $\left.w\left[n_{2}\right]\left(\mathbf{r}_{1}, \mathbf{r}_{2}\right)\right\}$ in the adiabatic approximation, one needs to go to the nonlinear regime and consider the next order terms $(\sim \gamma \Gamma)$ in the equations. To show the possibility of a biexcitonic solution, assume that the initial state includes a long-living exciton, $\gamma \Gamma \rightarrow \bar{\gamma} \Gamma$, where $\bar{\gamma}$ is the averaged "excitonic function" (see also Ref. 30, where a possibility to obtain double excitations in the adiabatic TDDFT was considered). Then, the second (biexcitonic) Eq. (17) acquires an additional term (at $G^{2}=0$ ), which results in the eigenenergy $2 E_{g}+2\left(h_{22}^{\gamma}-h_{11}^{\gamma}\right) \bar{\gamma}$ that corresponds to the biexcitonic solution in the case $\left(h_{22}^{\gamma}-h_{11}^{\gamma}\right) \bar{\gamma}<0$ and $2 E_{g}+2 \mid\left(h_{22}^{\gamma}\right.$ $\left.-h_{11}^{\gamma}\right) \bar{\gamma}|>2| F^{1} \mid=2 E_{\text {exc }}$. Possible excitations in the two-level case are illustrated in Fig. 1.

\section{TWO-BAND CASE}

The generalization to the many-electron case is straightforward. The equation for the biexciton energies (with zero center-of-mass momentum) in the two-band approximation has the following form:

$$
\begin{aligned}
& 0=\left[i \frac{\partial}{\partial t}-\varepsilon_{\mathbf{k}+\mathbf{q}}^{c}-\varepsilon_{\mathbf{k}^{\prime}}^{c}+\varepsilon_{\mathbf{k}}^{v}+\varepsilon_{\mathbf{k}^{\prime}+\mathbf{q}}^{v}\right] \Gamma_{\mathbf{k}+\mathbf{q}, \mathbf{k}^{\prime}, \mathbf{k}, \mathbf{k}^{\prime}+\mathbf{q}}^{c c v v}
\end{aligned}
$$

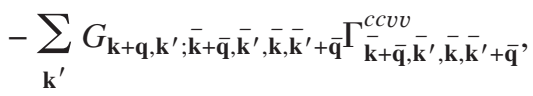

where 


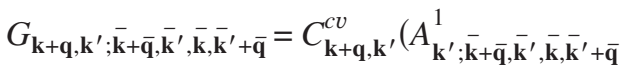

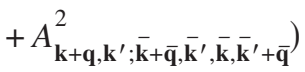

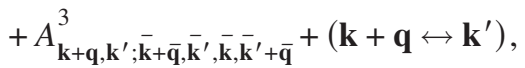

$$
\begin{aligned}
A_{\mathbf{k} ; \mathbf{k}_{1}, \mathbf{k}_{2}, \mathbf{k}_{3}, \mathbf{k}_{4}}^{1}= & \int d 1 d 2 d 3 \chi_{c \mathbf{k}}^{*}(1) \chi_{v \mathbf{k}}^{*}(1) \\
& \times g_{1}(1,2,3) \chi_{c \mathbf{k}_{1}}(2) \chi_{c \mathbf{k}_{2}}(3) \chi_{v \mathbf{k}_{3}}^{*}(2) \chi_{v \mathbf{k}_{4}}^{*}(3)
\end{aligned}
$$

$$
\begin{aligned}
A_{\mathbf{k}, \mathbf{k}^{\prime} ; \mathbf{k}_{1}, \mathbf{k}_{2}, \mathbf{k}_{3}, \mathbf{k}_{4}}^{2}= & \int d 1 d 2 d 3 d 4 \chi_{c \mathbf{k}}^{*}(1) \chi_{c \mathbf{k}^{\prime}}^{*}(2) \chi_{c \mathbf{k}}(1) \chi_{v \mathbf{k}^{\prime}}(2) \\
& \times g_{2}(1,2,3,4) \chi_{c \mathbf{k}_{1}}(3) \chi_{c \mathbf{k}_{2}}(4) \chi_{v \mathbf{k}_{3}}^{*}(3) \chi_{v \mathbf{k}_{4}}^{*}(4),
\end{aligned}
$$

$$
A_{\mathbf{k}, \mathbf{k}^{\prime} ; \mathbf{k}_{1}, \mathbf{k}_{2}, \mathbf{k}_{3}, \mathbf{k}_{4}}^{3}=\frac{1}{2} A_{\mathbf{k}, \mathbf{k}^{\prime} ; \mathbf{k}_{1}, \mathbf{k}_{2}, \mathbf{k}_{3}, \mathbf{k}_{4}}^{2}\left[\chi_{c \mathbf{k}}\left(\mathbf{r}_{1}\right) \rightarrow \chi_{v \mathbf{k}}\left(\mathbf{r}_{2}\right)\right],
$$

and $g_{1}\left(\mathbf{r}, \mathbf{r}_{1}, \mathbf{r}_{2}\right)=\frac{\delta V_{x c}(\mathbf{r})}{\delta n\left(\mathbf{r}_{1}, \mathbf{r}_{2}\right)}$ and $g_{2}\left(\mathbf{r}_{1}, \mathbf{r}_{2}, \mathbf{r}_{3}, \mathbf{r}_{4}\right)=\frac{\delta w\left(\mathbf{r}_{1}, \mathbf{r}_{2}\right)}{\delta n\left(\mathbf{r}_{3}, \mathbf{r}_{4}\right)}$ are two-particle density kernels. Similar to the excitonic case, Eq. (22) is the momentum representation version of the Schrödinger equation for two electrons and two holes, ${ }^{31}$ where the matrix elements $G_{\mathbf{k}+\mathbf{q}, \mathbf{k}^{\prime} ; \overline{\mathbf{k}}+\overline{\mathbf{q}}, \overline{\mathbf{k}}^{\prime}, \overline{\mathbf{k}}, \overline{\mathbf{k}}^{\prime}+\overline{\mathbf{q}}}$ correspond to an integral interparticle (in general, four-body) interaction.

To solve Eq. (22), we expand the biexcitonic function in terms of the complete set of the excitonic functions $\gamma_{n, \mathbf{k}, \mathbf{q}}^{c v}$ with eigenenergies $E_{n, \mathbf{q}}$ ( $n$ is the number of the bound state), which can be found from the solution of Eq. (15), and antisymmetrize it with respect to interchange of holes and electrons, in order to satisfy the Pauli principle. Then the biexcitonic functions can be expressed in the following form:

$$
\begin{aligned}
\widetilde{\Gamma}_{\mathbf{k}+\mathbf{q}, \mathbf{k}^{\prime}, \mathbf{k}, \mathbf{k}^{\prime}+\mathbf{q}}^{c c^{\prime} v^{\prime}}= & \sum_{n, m}\left[\gamma_{n, \mathbf{k}+\mathbf{q}, \mathbf{q}}^{v} \gamma_{m, \mathbf{k}^{\prime}+\mathbf{q},-\mathbf{q}}^{v^{\prime}} b_{n m, \mathbf{q}}^{ \pm}\right. \\
& \left.\mp \gamma_{n, \mathbf{k}^{\prime}, \mathbf{k}^{\prime}-\mathbf{k}}^{v} \gamma_{m, \mathbf{k}+\mathbf{q}, \mathbf{k}-\mathbf{k}^{\prime}}^{v^{\prime}} b_{n m, \mathbf{k}^{\prime}-\mathbf{k}}^{ \pm}\right],
\end{aligned}
$$

where \pm correspond to two possible states of biexcitons, singlet (-) and triplet $(+)$, with respect to two-electron spins. ${ }^{26}$ In this equation and below we omit the conduction index superscript in the excitonic wave functions since we consider the case of one spin-degenerate conduction band and the excitonic wave function can be completely defined by the hole (valence) index. Thus the problem is reduced to finding the matrix elements that enter into Eq. (22). Using the orthogonality of the excitonic eigenfunctions, one finds the equation for the biexcitonic eigenvectors and the corresponding eigenenergies, similar to Eq. (15),

$$
\begin{aligned}
& \sum_{n^{\prime}, m^{\prime}, \mathbf{q}^{\prime}}\left[\left(\omega-E_{n \mathbf{q}}-E_{m \mathbf{q}}\right) \delta_{n n^{\prime}} \delta_{m m^{\prime}} \delta_{\mathbf{q q}^{\prime}}-H_{n m, n^{\prime} m^{\prime}, \mathbf{q} \mathbf{q}^{\prime}}^{ \pm}\right] b_{n^{\prime} m^{\prime}, \mathbf{q}^{\prime}}^{ \pm} \\
& \quad=0
\end{aligned}
$$

$$
\begin{gathered}
H_{n m, n^{\prime} m^{\prime}, \mathbf{q} \mathbf{q}^{\prime}}^{ \pm}=\left([1 \mp \hat{S}]^{-1} \hat{W}^{ \pm}\right)_{n m, n^{\prime} m^{\prime}, \mathbf{q} \mathbf{q}^{\prime}}^{ \pm}, \\
\hat{S}_{n m, n^{\prime} m^{\prime}, \mathbf{q} \mathbf{q}^{\prime}}=\sum_{\mathbf{k}} \gamma_{n, \mathbf{k}+\mathbf{q}, \mathbf{q}}^{v} \gamma_{m, \mathbf{k}+\mathbf{q}^{\prime},-\mathbf{q}}^{v^{\prime}} \gamma_{n^{\prime}, \mathbf{k}+\mathbf{q}^{\prime}, \mathbf{q}^{\prime}}^{v} \gamma_{m^{\prime}, \mathbf{k}+\mathbf{q},-\mathbf{q}^{\prime}}^{v^{\prime}},
\end{gathered}
$$

and

$$
\begin{aligned}
& \hat{W}^{ \pm}=-\delta_{m m^{\prime}} \delta_{\mathbf{q q} \mathbf{q}^{\prime}} \sum_{\mathbf{k}, \mathbf{k}^{\prime}} \gamma_{n, \mathbf{k}+\mathbf{q}, \mathbf{q}}^{v} F_{\mathbf{k}, \mathbf{k}^{\prime}} \gamma_{n^{\prime}, \mathbf{k}+\mathbf{q}^{\prime}, \mathbf{q}^{\prime}}^{v} \\
& -\delta_{n n^{\prime}} \delta_{\mathbf{q q} \mathbf{q}^{\prime}} \sum_{\mathbf{k}, \mathbf{k}^{\prime}} \gamma_{m, \mathbf{k},-\mathbf{q}}^{v^{\prime}} F_{\mathbf{k}, \mathbf{k}^{\prime}} \gamma_{m^{\prime}, \mathbf{k}^{\prime},-\mathbf{q}}^{v^{\prime}} \\
& \pm \sum_{\mathbf{k}, \mathbf{k}^{\prime}} F_{\mathbf{k}, \mathbf{k}^{\prime}}^{*}\left(\gamma_{n, \mathbf{k}^{\prime}+\mathbf{q}, \mathbf{q}}^{v^{*}} \gamma_{m, \mathbf{k}+\mathbf{q}^{\prime},-\mathbf{q}}^{v^{\prime} *} \gamma_{n^{\prime}, \mathbf{k}+\mathbf{q}^{\prime}, \mathbf{q}^{\prime}}^{v} \gamma_{m^{\prime}, \mathbf{k}+\mathbf{q}^{\prime},-\mathbf{q}}^{v^{\prime}}\right. \\
& +\gamma_{m, \mathbf{k}^{\prime},-\mathbf{q}}^{v^{*}} \gamma_{n, \mathbf{k}-\mathbf{q}^{\prime}+\mathbf{q}, \mathbf{q}}^{v^{\prime} *} \gamma_{n^{\prime}, \mathbf{k}, \mathbf{q}^{\prime}}^{\left.v_{m^{\prime}, \mathbf{k}-\mathbf{q}^{\prime}+\mathbf{q},-\mathbf{q}^{\prime}}\right)} \gamma^{v^{\prime}}
\end{aligned}
$$

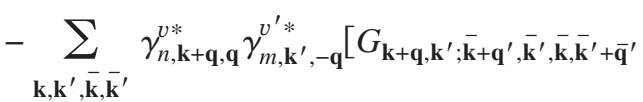

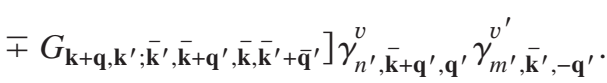

Equation (28) formally resembles Eq. (15) for excitons, with the exciton eigenenergies used instead of the bare singleelectron energies. The effective exciton-exciton attraction $H_{n m, n^{\prime} m^{\prime}, \mathbf{q q}^{\prime}}^{ \pm}$is defined by the matrix elements of the kernels $g_{1}\left(\mathbf{r}_{1}, \mathbf{r}_{2}, \mathbf{r}_{3}\right)$ and $g_{2}\left(\mathbf{r}_{1}, \mathbf{r}_{2}, \mathbf{r}_{3}, \mathbf{r}_{4}\right)$.

To test the formalism, we consider the simple case of a single biexciton in one-exciton level approximation, $n=m$ $=1$ (see also Fig. 1). We have obtained the solution of this equation using simple model kernels: the local kernel (1) to generate the excitonic states, and the following local twoparticle kernels for biexcitons:

$$
g_{1}^{\text {local }}\left(\mathbf{r}, \mathbf{r}_{1}, \mathbf{r}_{2}\right)=-C_{0} A_{1} \delta\left(\mathbf{r}-\mathbf{r}_{1}\right) \delta\left(\mathbf{r}-\mathbf{r}_{2}\right)
$$

[which includes the averaged element $C_{0}$ of the one-electron excited density-matrix component $C^{c v}$, see Eqs. (22)-(26)] and

$$
g_{2}^{\text {local }}\left(\mathbf{r}, \mathbf{r}^{\prime}, \mathbf{r}_{1}, \mathbf{r}_{2}\right)=-A_{2} \delta\left(\mathbf{r}-\mathbf{r}^{\prime}\right) \delta\left(\mathbf{r}-\mathbf{r}_{1}\right) \delta\left(\mathbf{r}-\mathbf{r}_{2}\right) .
$$

The kernels (32) and (33) can be viewed as constituting a "contact biexciton" model, in analogy with the contact exciton model defined by the XC kernel (1).

Results for the electron eigenenergies and eigenfunctions of several semiconductors were obtained by using the VASP 4.6 code $^{32}$ with GGA-projector augmented wave potentials and a $350 \mathrm{eV}$ energy cutoff. We approximated the NO functions by the corresponding Kohn-Sham single-particle wave functions, which can be considered as a good approximation when the correlations are not too strong. We find that with the effective local kernels $f_{\mathrm{XC}}^{\text {local }}, g_{1}^{\text {local }}$, and $g_{2}^{\text {local }}$ one can reproduce the experimental biexcitonic binding energies with a suitable choice of the parameters $A_{0}, A_{1}$, and $A_{2}$ (see Table I).

Let us now come back to the question how biexcitons can, in principle, be described within TDDFT. A closely related problem was studied by Maitra et al. for the case of finite 
TABLE I. Excitonic and biexcitonic binding energies $E_{b}^{\mathrm{exc}}$ and $E_{b}^{\mathrm{biexc}}$ for some semiconductors (in millielectron volt), calculated with the TDDMFT formalism. The parameters $A_{0}, A_{1}$, and $A_{2}$ which determine the model interaction kernels (1), (32), and (33) have been adjusted so that the calculations reproduce the experimental binding energies for excitons (see Refs. 33-36) and biexcitons (see Refs. 35 and 37-39).

\begin{tabular}{cccccc}
\hline \hline & $A_{0}$ & $E_{b}^{\text {exc }}$ & $C_{0} A_{1} / \Omega^{2}$ & $A_{2} / \Omega^{3}$ & $E_{b}^{\text {biexc }}$ \\
\hline $\mathrm{ZnO}$ & 290 & 60 & 1.82 & 101 & 15 \\
$\mathrm{CdS}$ & 308 & 28 & 0.022 & 0.64 & 5.7 \\
$\mathrm{CuCl}$ & 20.7 & 190 & 1.97 & 37.2 & 32 \\
$\mathrm{CuBr}$ & 20.9 & 110 & 2.0 & 11.5 & 25 \\
\hline \hline
\end{tabular}

systems, ${ }^{27}$ and they came up with a simple way (called "dressed" TDDFT) to construct a nonadiabatic $f_{\mathrm{XC}}(\omega)$ to generate those excitation that are missing if a frequencyindependent XC kernel is used. The idea is to consider a minimal subspace consisting of excited-state Kohn-Sham wave functions associated with quasidegenerate single and double excitations, and then diagonalize the full Hamiltonian in this subspace. This allows one to construct an explicitly frequency-dependent expression of $f_{\mathrm{XC}}(\omega)$ which is then used to recalculate the excitation energies. Applications to trans-polyenes demonstrated the potential of the method. ${ }^{28}$

To adapt this approach for the description of biexcitons in TDDFT, one would need to recast the approach of Maitra et $a l .{ }^{27}$ into momentum space, starting from Eqs. (15) and (16). A possible way to proceed would be to try to construct explicitly frequency-dependent matrix elements $F_{\mathbf{k} \mathbf{k}^{\prime}}(\omega)$ of the nonadiabatic XC kernel in terms of the adiabatic potential $w[n]\left(\mathbf{r}_{1}, \mathbf{r}_{2}\right)$ (or, rather, the two-particle kernels $g_{1}$ and $g_{2}$ ) which produces biexcitonic states. In this way, the exciton Eq. (15) would produce additional solutions, corresponding to biexcitonic binding energies, and would also result in (presumably small) shifts of the original excitonic energies due to the exciton-biexciton coupling that is now taken into account. Whether or not such a TDDFT procedure will be practical remains a question for future investigation.

\section{CONCLUSION}

In this paper we have formulated a TDDMFT approach to study biexcitonic effects. We have derived the TDDMFT version of the Schrödinger equation for biexcitons in terms of the two-particle density-matrix elements in the two-band approximation. We have solved this equation in the case of several semiconductors by using phenomenological twoelectron interaction kernels, thereby defining a contact biex- citon model. With this model one can reproduce the lowest biexcitonic binding energies by using proper kernel parameters. To obtain biexcitonic states within the single-particle TDDFT approach, one would either need to use a frequencydependent XC kernel or consider the nonlinear regime. Generalization for the case of bound states with larger number of particles is, in principle, straightforward.

There are several advantages of this simplified formalism for biexcitons comparing to other approaches: (1) it can be adapted for use in the real-time domain in a straightforward manner; (2) physical transparency of the method, in particular, the effective TDDFT electron-hole and exciton-exciton interactions are directly related to the interaction kernels, which may allow one to make simple estimations of the possibility to produce bound states with given TDDFT kernels; (3) it may allow one to construct the nonadiabatic KohnSham XC kernel from the phenomenological adiabatic twoparticle density kernel, which may shed some light on the general requirements on the nonadiabatic $f_{\mathrm{XC}}(\omega)$ necessary to produce biexcitons and other higher-order coupled states with TDDFT.

Examination of ultrafast processes and higher-order correlation effects, including the excitonic and biexcitonic transport, in semiconductor nanostructures and organic molecules is underway.

\section{ACKNOWLEDGMENTS}

This work was supported in part by DOE under Grant No. DE-FG02-07ER15842 (V.T. and T.S.R.) and NSF under Grants No. ECCS 072551 and No. ECCS-0901784, AFOSR under Grant No. FA9550-09-1-0450, and through the DARPA/MTO Young Faculty Award No. HR0011-08-1-0059 (M.N.L.). C.A.U. acknowledges support from NSF under Grants No. DMR-0553485 and No. DMR-1005651.

\footnotetext{
*Corresponding author; mleuenbe@mail.ucf.edu

${ }^{1}$ G. Onida, L. Reining, and A. Rubio, Rev. Mod. Phys. 74, 601 (2002).

${ }^{2}$ F. Rossi and T. Kuhn, Rev. Mod. Phys. 74, 895 (2002).

${ }^{3}$ M. Erementchouk, M. N. Leuenberger, and L. J. Sham, Phys. Rev. B 76, 115307 (2007).

${ }^{4}$ M. Erementchouk, M. N. Leuenberger, and L. J. Sham, Phys.
}

Rev. B 79, 085307 (2009).

${ }^{5}$ V. Z. Tronciu and R. A. Abram, Phys. Rev. E 65, 026616 (2002).

${ }^{6}$ M. Erementchouk and M. N. Leuenberger, Phys. Rev. B 81, 195308 (2010).

${ }^{7}$ X. Li, Ya. Wu, D. Steel, D. Gammon, T. H. Stievater, D. S. Katzer, D. Park, C. Piermarocchi, and L. J. Sham, Science 301, 809 (2003). 
${ }^{8} \mathrm{H}$. Haug and S. W. Koch, Quantum Theory of the Optical and Electronic Properties of Semiconductors (World Scientific, Singapore, 2001).

${ }^{9}$ L. P. Kadanoff and G. Baym, Quantum Statistical Mechanics (Benjamin, New York, 1962).

${ }^{10}$ L. V. Keldysh, Zh. Eksp. Teor. Fiz. 47, 1515 (1964) [Sov. Phys. JETP 20, 1018 (1965)].

${ }^{11}$ D. S. Chemla and J. Shah, Nature (London) 411, 549 (2001).

${ }^{12}$ E. Runge and E. K. U. Gross, Phys. Rev. Lett. 52, 997 (1984).

${ }^{13}$ S. Botti, A. Schindlmayr, R. Del Sole, and L. Reining, Rep. Prog. Phys. 70, 357 (2007).

${ }^{14}$ Y. H. Kim and A. Görling, Phys. Rev. Lett. 89, 096402 (2002); Phys. Rev. B 66, 035114 (2002).

${ }^{15}$ V. Turkowski and C. A. Ullrich, Phys. Rev. B 77, 075204 (2008).

${ }^{16}$ V. Turkowski, A. Leonardo, and C. A. Ullrich, Phys. Rev. B 79, 233201 (2009).

${ }^{17}$ F. Sottile, K. Karlsson, L. Reining, and F. Aryasetiawan, Phys. Rev. B 68, 205112 (2003).

${ }^{18}$ J. E. Rowe and D. E. Aspnes, Phys. Rev. Lett. 25, 162 (1970); 25, 979(E) (1970).

${ }^{19}$ R. M. Martin, J. A. van Vechten, J. E. Rowe, and D. E. Aspnes, Phys. Rev. B 6, 2500 (1972).

${ }^{20}$ S. Botti, F. Sottile, N. Vast, V. Olevano, L. Reining, H.-C. Weissker, A. Rubio, G. Onida, R. Del Sole, and R. W. Godby, Phys. Rev. B 69, 155112 (2004).

${ }^{21}$ S. Botti, A. Fourreau, F. Nguyen, Y.-O. Renault, F. Sottile, and L. Reining, Phys. Rev. B 72, 125203 (2005).

${ }^{22}$ S. Sagmeister and C. Ambrosch-Draxl, Phys. Chem. Chem. Phys. 11, 4451 (2009).

${ }^{23}$ K. Pernal, O. Gritsenko, and E. J. Baerends, Phys. Rev. A 75, 012506 (2007).

${ }^{24}$ K. J. H. Giesbertz, E. J. Baerends, and O. V. Gritsenko, Phys. Rev. Lett. 101, 033004 (2008).
${ }^{25}$ K. J. H. Giesbertz, K. Pernal, O. V. Gritsenko, and E. J. Baerends, J. Chem. Phys. 130, 114104 (2009).

${ }^{26} \mathrm{~W}$. Schäfer and M. Wegener, Semiconductor Optics and Transport Phenomena (Springer, Berlin, 2002).

${ }^{27}$ N. T. Maitra, F. Zhang, R. J. Cave, and K. Burke, J. Chem. Phys. 120, 5932 (2004).

${ }^{28}$ R. J. Cave, F. Zhang, N. T. Maitra, and K. Burke, Chem. Phys. Lett. 389, 39 (2004).

${ }^{29}$ T. L. Gilbert, Phys. Rev. B 12, 2111 (1975).

${ }^{30}$ C. M. Isborn and X. Li, J. Chem. Phys. 129, 204107 (2008).

${ }^{31}$ R. R. Sharma, Phys. Rev. 170, 770 (1968); P. H. Handel, Phys. Rev. B 7, 5183 (1973); J. J. Forney, A. Quattropani, and F. Bassani, II Nuovo Cimento B 22, 153 (1974).

${ }^{32} \mathrm{G}$. Kresse, J. Hafner, and M. Wegener, Computer Code VASP Guide (University of Vienna, Austria, 2003).

${ }^{33}$ S. Adachi, K. Hazu, T. Sota, S. F. Chichibu, G. Cantwell, D. B. Eason, D. C. Reynolds, and C. W. Litton, Semicond. Sci. Technol. 19, S276 (2004).

${ }^{34}$ M. A. Jakobson, V. D. Kagan, R. P. Seisyan, and E. V. Goncharova, J. Cryst. Growth 138, 225 (1994).

${ }^{35}$ Excitonic Processes in Solids, Springer Series of Solid-State Sciences, Vol. 60, edited by M. Ueta, H. Kanzaki, K. Kobayashi, Y. Toyozawa, and E. Hanamura (Springer, Berlin, 1986).

${ }^{36}$ O. V. Gogolin, E. G. Tsitsishvili, J. L. Deiss, C. Klingshirn, and V. E. Solomko, JETP Lett. 34, 312 (1981).

${ }^{37}$ H. J. Ko, Y. F. Chen, T. Yao, K. Miyajima, A. Yamamoto, and T. Goto, Appl. Phys. Lett. 77, 537 (2000).

${ }^{38}$ R. Baumert, I. Broser, and K. Buschick, IEEE J. Quantum Electron. 22, 1539 (1986); S. Shionoya, H. Saito, E. Hanamura, and O. Akimoto, Solid State Commun. 12, 223 (1973); H. Saito, S. Shionoya, and E. Hanamura, ibid. 12, 227 (1973).

${ }^{39}$ Y. Masumoto, S. Okamoto, and S. Katayanagi, Phys. Rev. B 50, 18658 (1994). 